\title{
Strategien zur Umsetzung einer hochwasserangepassten Raumnutzung
}

Zusammenfassung: Der vorliegende Artikel vereint vier Beiträge aus dem FloodRisk II Arbeitspaket Raumplanung, die sich der Sicherung und hochwasserangepassten Nutzung flussnaher unbebauter Räume widmen. Die Autoren greifen Defizite bestehender Regelungen und Instrumente von Raumplanung und Schutzwasserwirtschaft auf und legen umsetzungsorientierte Strategien für eine Raumnutzung vor, die sich an der Zielvorstellung einer Reduktion von Hochwasserrisiken orientiert. Die Beiträge nehmen dabei auf die Umsetzung einer gemeindeübergreifenden, fluss- oder einzugsgebietsbezogenen Hochwasservorsorge und den damit verbundenen Ausgleich von Kosten und Nutzen des Hochwasserschutzes zwischen Ober- und Unterliegern ebenso Bezug wie auf eine verbesserte Koordination von Schutzwasserwirtschaft und Raumentwicklung und die Möglichkeiten einer hochwasserverträglichen landwirtschaftlichen Bewirtschaftung potentieller Hochwasserabflussund Rückhalteräume.

Strategies for the implementation of a land-use policy allowing for potential flood risks

Summary: This article unites four papers from the Federal Environmental Agency's FloodRisk II land-use planning work package which are intended to ensure the use of riverine areas without buildings with regard to potential flood risks. The authors pick up on the shortcomings of existing rules and instruments of regional planning and flood management, and submit strategies for introducing land-use policies aiming at reducing flood risks. This includes the implementation of a transcommunal flood-prevention scheme based on river systems or catchments, and the cost-benefit balance of flood protection between upper and lower riparians, as well as the improved coordination of flood-control management and regional development, together with the possibilities of agricultural use in keeping with the flood risk in potential flood plains and flood-retention areas.

\section{Einleitung}

Gewässernahe unbebaute Räume sind eine der wichtigsten Ressourcen für Hochwasserschutz und Gewässerentwicklung. Besonders im alpinen Raum sind sie aufgrund der topografischen Verhältnisse und der Nutzung für Siedlungs- und Verkehrszwecke nur noch sehr eingeschränkt verfügbar. Der Umgang mit der Inanspruchnahme von unbebautem Boden zählt zu den zentralen Anliegen der Raumplanung. Mit deren Instrumenten sollen Raumansprüche und Raumnutzungen entsprechend der Eignung der jeweiligen Standorte unter Vermeidung räumlicher Konflikte verteilt werden. Naturgefahren wie Hochwasserereignisse schränken die Eignung eines Standortes für Nutzungen wie Wohnen, Gewerbe, Industrie, Freizeit, Landwirtschaft und Verkehr erheblich ein. Die Raumplanung ist im Hinblick auf Naturgefahren in erster Linie präventiv wirksam, indem gefährdete Bereiche von baulichen Intensivnutzungen möglichst freigehalten werden. Das bestehende Raumplanungssystem in Österreich weist diesbezüglich einige Defizite auf bzw. bedarf es ergänzender Ansätze in jenen Bereichen, wo die Instrumente der Raumplanung nur wenig steuerungswirksam sind. Im thematisch breit ausgerichteten FloodRisk II Arbeitspaket Raumplanung wurden in diesem $\mathrm{Zu}$ sammenhang die regionale Dimension der Hochwasservorsorge in der Raumplanung, Ausgleichsmechanismen zwischen Oberund Unterliegern an Fließgewässern, die Abstimmung zwischen Schutzwasserwirtschaft und Raumplanung sowie die Rolle der Landwirtschaft im Hochwasserschutz in vier verschiedenen Forschungsprojekten aufgegriffen. Ansätze und Ergebnisse dieser Forschungsaktivitäten werden nachfolgend vorgestellt.

\section{Interkommunale Ansätze in der Hochwasservorsorge}

\subsection{Die regionale Dimension im Hochwasserschutz}

Dieser Abschnitt stellt ausgewählte Ergebnisse aus dem vom Bundesministerium für
Verkehr, Innovation und Technologie (BMVIT) beauftragten FloodRisk II-Teilprojekt „Praktische Umsetzung künftiger Strategien risikoarmer Raumnutzung - Absiedelung und Flächenwidmung" vor, das sich aus den Projektteilen „Absiedelung“ und „Interkommunale Kooperation“ zusammensetzt (vgl. Seher und Berger, 2009). Im Projektteil „Interkommunale Kooperation“ wurden mit Hilfe eines akteurs- und prozessorientierten Forschungsansatzes die Rahmenbedingungen gemeindeübergreifender Zusammenarbeit in der Hochwasservorsorge anhand der Fallstudie Hochwasserschutzverband Aist (Oberösterreich) sowie von Erkenntnissen aus anderen hochwasserbezogenen Kooperationsansätzen untersucht.

Hochwasser machen in der Regel nicht an Gemeindegrenzen halt. Die unterschiedliche Lage von Gemeinden am Flusslauf hat unterschiedliche Handlungsmöglichkeiten und einseitige $\mathrm{Ab}$ hängigkeiten zur Folge, die sich mit dem in der Fachliteratur (vgl. u.a. Heiland, 2002; Frerichs et. al., 2003) verwendeten Begriff Oberlieger-Unterlieger-Verhältnis charakterisieren lassen. Technische Schutzmaßnahmen und die Versiegelung potenzieller Retentionsflächen durch bauliche Nutzung sind als Einzelentscheidungen der Oberliegergemeinden rational, weil sie mit wirtschaftlichem Nutzen verbunden sind. Die negativen Auswirkungen gehen als externe Effekte aber zu Lasten anderer Gemeinden. Die regionale Dimension von Hochwasserereignissen erfordert regionales, d.h. gemeindeübergreifendes Handeln im Sinne einer einzugsgebiets- bzw. auf einzelne Flussabschnitte bezogenen Betrachtungsweise.

Die regionale Ebene wird aus ExpertInnensicht ${ }^{1}$ als für den Hochwasserschutz sehr bedeutsam angesehen. Diese Sichtweise spiegelt sich innerhalb der Raumplanung weder in den Raumordnungsgesetzen der Bundesländer noch in der Handlungspraxis der AkteurInnen wider.

So verfolgen etwa die Wasserrahmenrichtlinie und die Richtlinie über die Bewertung und das Management von Hochwasserrisiken (,,Hochwasserrichtlinie“) einen regionalen, fluss- oder einzugsgebietsbezogenen Ansatz. 
Während in Österreich Informationen zur Hochwassergefährdung auf regionaler Ebene vielfach zur Verfügung stehen, sind raumplanerische Festlegungen zur Reduktion von Hochwasserrisiken mit verpflichtendem Charakter auf der überörtlichen, d.h. der über die Gemeinde hinausgehenden Ebene, gegenwärtig nicht vorgesehen. Raumplanerische Festlegungen sind zwar nicht ausgeschlossen, zählen aber nicht zu den Kerninhalten der Regionalplanung, weder was überörtliche Bauverbote noch was die Ersichtlichmachung von Gefahrenbereichen anbelangt (vgl. Kanonier, 2005).

Ein fluss- oder einzugsgebietsbezogener Ansatz in der Hochwasservorsorge lässt sich vor dem Hintergrund der fehlenden Möglichkeiten verpflichtender regionalplanerischer Steuerung nur über die freiwillige Kooperation von Gemeinden realisieren. Welche Rahmenbedingungen für deren Implementierung erforderlich sind, soll im Folgenden dargestellt werden.

\subsection{Interkommunale Kooperation in der Hochwasservorsorge - Rahmenbedingungen}

Die interkommunale Kooperation in der Hochwasservorsorge bezeichnet eine institutionalisierte Zusammenarbeit von AkteurInnen und EntscheidungsträgerInnen aus zwei oder mehreren Gemeinden in einem Flusseinzugsgebiet mit dem Ziel der Verminderung von Hochwasserrisiken durch gemeinsame Umsetzung von räumlich wirksamen Maßnahmen. Aufgabenfelder von interkommunalen Kooperationen in der Hochwasservorsorge sind aktuell die Standortsuche für Retentionsräume mit überörtlicher Bedeutung sowie die Freihaltung überörtlich bedeutsamer Retentionsflächen.

Die Größe des Kooperationsgebietes sowie die Anzahl und die Rechtsform der potentiellen Mitglieder stellen neben den Inhalten und den Zielen einer Kooperation die wesentlichen Parameter für die Auswahl der Organisationsform dar. Oberlieger-Unterlieger-Vereinbarungen sind Steuerungsinhalte, die in der Regel keine „win-win-Situationen“ für die Planungsadressaten erwarten lassen. Für solche Steuerungsinhalte kommen nur formelle Kooperationen (z. B. Wasserverbände, Gemeindeverbände) mit entsprechenden rechtlich verankerten Kompetenzen in Frage. Mit dem hohen Institutionalisierungsgrad sind die Verbindlichkeit von Vereinbarungen, Sanktionsmöglichkeiten, die Außenwirk- samkeit und die Durchsetzungskraft der Kooperationen verbunden.

Interkommunale Initiativen in der Hochwasservorsorge sind „disaster driven“, d.h. sie erfordern Problemdruck durch Hochwasserschäden in jüngerer Vergangenheit und das daraus resultierende Problembewusstsein maßgeblicher kommunaler AkteurInnen. Wesentlich für die Entstehung dieser Gemeindekooperationen sind ferner die Initiative, das Engagement und die Überzeugungskraft von Einzelpersonen, die über die Fähigkeiten und Möglichkeiten verfügen, den Kooperationsprozess einzuleiten und aufrechtzuerhalten sowie die Unterstützung von (Landes)Politik und (Landes)Verwaltung. Gut aufbereitete fachliche Grundlagen, wie z. B. in Form von Regionalstudien, bilden die Basis für Inhalt und Organisation der Kooperation, den Finanzierungsbedarf und daraus resultierend die Verhandlungsgrundlage für die Aufteilung der Kosten.

Die Einigung über die Aufteilung der entstehenden Kosten stellt einen, wenn nicht den entscheidenden Aspekt für das Zustandekommen einer interkommunalen Kooperation in der Hochwasservorsorge dar. Die Beitragsschlüssel der in diesem Projekt untersuchten Kooperationen berücksichtigen die Hochwasserbetroffenheit und die Handlungsmöglichkeiten der beteiligten Gemeinden und enthalten in jeweils unterschiedlicher Zusammensetzung die folgenden Faktoren:

- die Gemeindeflächen im Einzugsgebiet mit abgestufter Bewertung nach Nutzungsarten

- das Schadenspotential in der Gemeinde

- die Schäden bei einem Referenzereignis innerhalb der Gemeinde

- die Rückhaltevolumina in der jeweiligen Gemeinde

- die Finanzkraft der Gemeinden.

Direkte Kompensations- oder Ausgleichsmaßnahmen zwischen Gemeinden sind gegenwärtig nicht umgesetzt. Ein Ansatz zum interkommunalen Ausgleich von Nutzen und Lasten der Hochwasservorsorge wird in Abschnitt 3 vorgestellt.

Als bedeutendste Aufgabe der Raumplanung im Zuge von gemeindeübergreifenden Ansätzen im Hochwasserschutz wird die Flächensicherung gesehen, d.h. die Freihaltung jener Flächen von baulichen Intensivnutzungen, die als Beckenstandorte oder für die fließende Retention vorgesehen sind. Für die Flächensicherung ist eine verpflichtende Berücksichtigung der Kooperationsver- einbarungen in der örtlichen Raumplanung der beteiligten Gemeinden erforderlich. Ist diese Möglichkeit gegeben, kann die Flächensicherung auch auf kommunaler Ebene über die örtlichen Entwicklungskonzepte und die Flächenwidmungspläne erfolgen.

\subsection{Fazit und Empfehlungen}

Interkommunale Kooperationen können unter den genannten Rahmenbedingungen eine effektive Möglichkeit einer einzugs- oder flussgebietsbezogenen Berücksichtigung von Hochwasserrisiken darstellen. Ausgehend von der Fallstudie Aist und den Erkenntnissen aus anderen hochwasserbezogenen Kooperationsansätzen können u.a. folgende Empfehlungen formuliert werden:

- Das zur Gründung einer Kooperation erforderliche Problembewusstsein nimmt mit zeitlichem Abstand zu einem Hochwasserereignis ab. AkteurInnen aus der Verwaltung und den Gemeinden wird empfohlen, dieses limitierte Zeitfenster zur Initiierung einer gemeindeübergreifenden Zusammenarbeit zu nützen.

- Gemeindekooperationen können nicht verordnet werden. Förderungen für Kooperationen können wesentliche Anstöße für eine interkommunale Kooperation in der Hochwasservorsorge darstellen.

- Die Akzeptanz von regional wirksamen Hochwasserschutzmaßnahmen hängt eng mit der Aufteilung der Kosten zusammen. Dazu sind Ausgleichsmechanismen zwischen Gemeinden mit hoher überörtlicher Schutzwirkung und solchen, die davon profitieren, weiterzuentwickeln.

- Da in Österreich gegenwärtig keine gesetzlichen Festlegungen zur Sicherung überörtlich bedeutsamer Retentionsflächen in der Regionalplanung implementiert sind, wird empfohlen, überörtlich bedeutsame Retentions- und Hochwasserabflussflächen bei der Festlegung von Siedlungsgrenzen und regionalen bzw. überörtlichen Grünzonen zu berücksichtigen.

Abschließend bleibt aber zu sagen, dass die interkommunale Kooperation in der Hochwasservorsorge die fehlende Berücksichtigung einer entsprechenden Flächenfreihaltung in der Regionalplanung nicht vollwertig ersetzt. Soll, wie in der EUHochwasserrichtlinie gefordert, Hochwasserrisikomanagement auf Einzugs- oder 
Flussgebietsebene präventiv ${ }^{2}$ und ohne unmittelbaren Anlassfall betrieben werden, sollte für eine verpflichtende Berücksichtigung des Hochwasserrisikos in der überörtlichen Raumplanung die Möglichkeit zur Verordnung spezifischer Widmungskategorien (z. B. Retentionsfläche mit überörtlicher Bedeutung) geschaffen werden.

\section{Kompensation von Retentions-} flächen: ein Modell zur wirtschaftlichen Entschädigung und zum interkommunalen Lastenausgleich

\subsection{Zielsetzung}

Im Rahmen des vom Amt der Niederösterreichischen Landesregierung, Abteilung Wasserbau beauftragten FloodRisk II Projektes „Integrative Gewässerentwicklung und interkommunaler Lastenausgleich, Flächenbedarf und -ausgleich für Hochwasserschutz, Siedlungsentwicklung und Ökologie" wurden methodische Grundlagen zur Förderung einer integrativen Flächennutzung über längere Flussabschnitte entwickelt. Das Projekt wurde von einem interdisziplinären Team, bestehend aus ezb - TB Eberstaller, DonauConsult Zottl \& Erber, dem Institut für Hydrobiologie und Gewässermanagement sowie dem Institut für Raumplanung und Ländliche Neuordnung, beide Universität für Bodenkultur Wien, bearbeitet (vgl. Eberstaller et al., 2009).

Überflutungsvolumen, das aufgrund von Hochwasserschutzmaßnahmen für höherwertig genutztes Gebiet verloren geht, muss entsprechend den aktuellen Richtlinien der Wasserbauverwaltung (RIWA-T) durch Schaffung bzw. Vergrößerung des Abflussvolumens an anderer Stelle hydrologisch gleichwertig kompensiert werden. Kompensationsmaßnahmen finden normalerweise in unmittelbarer Nähe der betroffenen Fläche innerhalb einer Gemeinde statt. Gelingt dies aufgrund beengter räumlicher Verhältnisse nicht, müssen die Kompensationsmaßnahmen in Nachbargemeinden erfolgen, wodurch das räumliche und wirtschaftliche Entwicklungspotential dieser Gemeinden eingeschränkt wird.

Als Basis für Verhandlungen zwischen Ober- und Unterliegergemeinden wurde ein Modell für ein Kompensationsangebot entwickelt. Dieses Modell soll den

\footnotetext{
2 Die Schwerpunkte der Hochwasserrisikomanagementpläne liegen auf Vermeidung, Schutz und Vorsorge (Art. 7, Abs. 3 Hochwasserrichtlinie).
}

Flächen- bzw. Lastenausgleich zwischen einzelnen Flussabschnitten (Gemeinden) anhand von „RetentionsflächenÄquivalenten“ ermöglichen, die als Basis für den ökonomischen Ausgleich zwischen den Gemeinden dienen. Die Bewertung der Retentionsflächen erfolgt hierbei sowohl nach sozioökonomischen Gesichtspunkten, als auch in wasserwirtschaftlicher Hinsicht. Durch einen pragmatischen Ansatz sollen mit möglichst geringem Berechungsaufwand alle relevanten Parameter einbezogen werden, da eine nachvollziehbare Berechnung des Kompensationsangebotes eine wesentliche Grundlage für die Akzeptanz des Angebotes zwischen den Gemeinden ist. Durch die fiktive Anwendung an drei Beispielsgemeinden an der Traisen in Niederösterreich wurden Praktikabilität und Plausibilität des Modells getestet.

\subsection{Hydrologisch/hydraulische und ökonomische Kennwerte des Modells}

Die Basis für die Anwendung des Modells ist die Kenntnis folgender hydrologischer und hydraulischer Parameter:

- Auswirkungen des durch geplante Verbauungsmaßnahmen verloren gehenden Retentionsvolumens (Wellenbeschleunigung, Scheitelerhöhung)

- Potentielle Wirkung von retentionserhöhenden Maßnahmen auf noch vorhandenen freien Flächen flussauf (z. B. Strukturierung des Flussbetts, Aufweitung, Erhöhung der Rauigkeit im Vorland)

- Fläche, die zur hydrologischen Kompensation des verloren gegangenen Retentionsvolumens nötig ist. Diese Fläche ist ein wesentlicher Eingangsparameter des Kompensationsmodells.

Das Kompensationsmodell geht davon aus, dass eine Gemeinde durch die Bebauung einer durch Deiche hochwasserfrei gewordenen Fläche einen finanziellen Gewinn erzielen kann. Eine Oberliegergemeinde hat durch eine allfällige Bereitstellung zusätzlicher Retentionsflächen langfristig einen finanziellen Verlust, da diese Flächen für die Erzielung monetärer Vorteile nicht oder nur in geringerem Ausmaß zur Verfügung stehen. Grundlegende Überlegungen in Bezug auf ökonomische Instrumente zum Lasten-Nutzen-Ausgleich im Hochwasserschutz sowie ein Berechnungsbeispiel finden sich bei Heiland (2002).
Diese Überlegungen bildeten den Ausgangspunkt für die Erstellung des ökonomischen Ausgleichsmodells.

Das Kompensationsangebot (d.h. die Ausgleichszahlung) einer Unterliegergemeinde an eine Gemeinde im Oberlauf ergibt sich aus nachfolgend dargestellter Formel, wobei folgende Faktoren berücksichtigt werden:

- Kosten in der Oberliegergemeinde

- Herstellungskosten (Planungskosten, Baukosten etc.) ... BK

- Grunderwerbs- bzw. Entschädigungskosten für den Grundeigentümer ... GK

- Transaktionskosten ... TK

- Opportunitätskosten; diese entsprechen dem Nutzungsentgang durch nicht verwirklichte, wertsteigernde Nutzungen (z. B. Aufschließung eines Gewerbegebiets) ... OK

- Nutzen in der Oberliegergemeinde

- Nutzen der Oberliegergemeinde durch die Kompensationsmaßnahme (z.B. Revitalisierung von Auen, Schaffung von Erholungsgebieten) ... No

- Opportunitätserlöse: Kosten, die der Oberliegergemeinde im Zusammenhang mit nicht realisierten Nutzungen nicht entstehen (z.B. Aufschließungskosten) ... OE

Zusätzlich wird mit einem Faktor „p“ die Realisierungswahrscheinlichkeit der zukünftig verhinderten höherwertigen Nutzung auf den Kompensations-Retentionsflächen in der Oberliegergemeinde berücksichtigt (mit $0<\mathrm{p}<1)$. Der Faktor „V“ bildet jene Vorteile ab, die für den Oberlieger durch die Realisierung einer höherwertigen Nutzung im Unterlauf, etwa über Pendlerverflechtungen oder zentralörtliche Funktionen der Unterliegergemeinde, entstehen. Die „Netto-Opportunitätskosten“ (OK *p - OE *p) werden demgemäß mit dem Faktor "v“ abgemindert $(0<\mathrm{v}<1)$. Damit errechnet sich das Kompensationsangebot KA wie folgt:

$\mathrm{KA}=\mathrm{BK}+\mathrm{GK}+\mathrm{TK}+\left(\mathrm{OK}^{*} \mathrm{p}-\mathrm{OE}^{*} \mathrm{p}\right)^{*} \mathrm{v}-\mathrm{No}$

Die Realisierungswahrscheinlichkeit der Ausgleichsmaßnahme hängt vom Verhältnis zwischen dem Kompensationsangebot und dem Nutzen für die Unterliegergemeinde $a b$. Je höher der Nutzen im Vergleich zum Kompensationsanbot ist, desto höher ist die Realisierungswahrscheinlichkeit der Ausgleichsmaßnahme. 


\subsection{Schlussfolgerungen und Empfehlungen}

Mit dem vorgeschlagenen Modell kann die Höhe des Kompensationsangebotes als Basis für Verhandlungen zwischen Ober- und Unterliegergemeinden zur Bereitstellung von im Rahmen der RIWA-T geforderten Kompensationsflächen ermittelt werden. Das vorgeschlagene Kompensationsmodell bietet die Möglichkeit, Gemeinden mit geringem wirtschaftlichem Entwicklungspotential für die Bereitstellung von Überflutungsflächen zu entschädigen. Gleichzeitig können Unterliegergemeinden ein hohes wirtschaftliches Entwicklungspotential nutzen. Dabei ergeben sich folgende grundsätzliche Überlegungen zu den Grenzen und Möglichkeiten der Kompensation von Überflutungsflächen:

- Die Kompensation von Überflutungsflächen sollte nicht isoliert für einzelne Maßnahmen, sondern nur in Abstimmung mit einem Entwicklungsplan für das gesamte Einzugsgebiet erfolgen.

- Wesentlich ist die Kenntnis der hydrologischen, der hydraulischen und der ökologischen Bedeutung noch vorhandener Überflutungsflächen.

- Die Abdämmung von Überflutungsflächen ist gemäß RIWA-T nur dann förderfähig, wenn die zu schützenden Flächen bereits höherwertig genutzt, dem Lückenschluss derartiger Flächen dienen oder zumindest höherwertig gewidmet sind.

- Abschnitte, wo Überflutungsflächen aus dem Abflussprofil genommen werden, erfordern ev. trotzdem Maßnahmen, die die Erreichung der ökologischen Ziele der EU-Wasserrahmenrichtlinie ermöglichen.

\section{Der „SREP Kärnten“ - Ein Modell zur Flächensicherung an Flüssen}

\subsection{Einleitung}

Raumplanerische Strategien gewinnen im integralen Risikomanagement zunehmend an Bedeutung. Viele Hochwasserschutzmaßnahmen können nur realisiert werden, wenn der erforderliche Raumbedarf mit anderen Nutzungen abgestimmt und langfristig gesichert ist. Der Schutzwasserwirtschaftliche RaumEntwicklungsPlan (kurz: SREP), der in Kärnten am Beispiel von zwei Flüssen (Möll und Gurk) pilothaft entwickelt wurde, setzt hier an. Flächen für künftige schutzwasserwirt- schaftliche Vorhaben sollen durch konsensuale Abstimmung der Nutzungsinteressen nachhaltig zur Verfügung stehen.

\subsection{Ablauf und Planungsschritte}

In einem ersten Schritt wird der Flächenbedarf von (Schutz)Wasserwirtschaft und Raumplanung erhoben und jeweils vier Anspruchsklassen (von gering bis sehr hoch) zugeordnet. Durch die Überlagerung der aktuellen und geplanten Flächenansprüche der Gemeinden mit den Flächenansprüchen der Schutzwasserwirtschaft mit Hilfe eines Geografischen Informationssystems (GIS) werden jene (Konflikt-)Flächen sichtbar, auf die sowohl die Raumentwicklung als auch die (Schutz)Wasserwirtschaft Anspruch erheben (Schritt 2). Im Zuge der Konfliktlösung (Schritt 3) wird zunächst anhand eines standardisierten Schemas eine grobe Zuordnung der Flächen vorgenommen. Wo gleichzeitig starke Ansprüche der (Schutz)Wasserwirtschaft und Raumentwicklung aufeinanderprallen, versagt die automatische Zuteilung. Hier wird in ei- nem zweiten Teilschritt „händisch“ im Rahmen eines Kommunikationsprozesses zugeordnet und bereinigt. Dafür wurden eigene Empfehlungen und Regeln erstellt. Am Ende des Prozesses (Schritt 4) steht der Schutzwasserwirtschaftliche Raumentwicklungsplan (SREP). Darin sind die von der (Schutz)-Wasserwirtschaft benötigten und mit der Raumentwicklung abgestimmten „Bedarfsflächen“ räumlich ausgewiesen. An die „Bedarfsflächen“ sind zugleich Handlungsempfehlungen geknüpft. (Abb. 1)

\subsection{Gefahrenzonenplan Flussbau und SREP}

Derzeit erfolgt die Sicherung des notwendigen Flussraums v. a. über den Gefahrenzonenplan des Flussbaus. Dieser analysiert den Ist-Zustand der Gefahrensituation, ist also kein Planungsinstrument, sondern ein Fachgutachten, das kaum eine zeitliche und inhaltliche Abstimmung zwischen Wasserwirtschaft und Raumplanung vorsieht. Dem gegenüber fließen in den SREP schutzwasserwirtschaftliche,

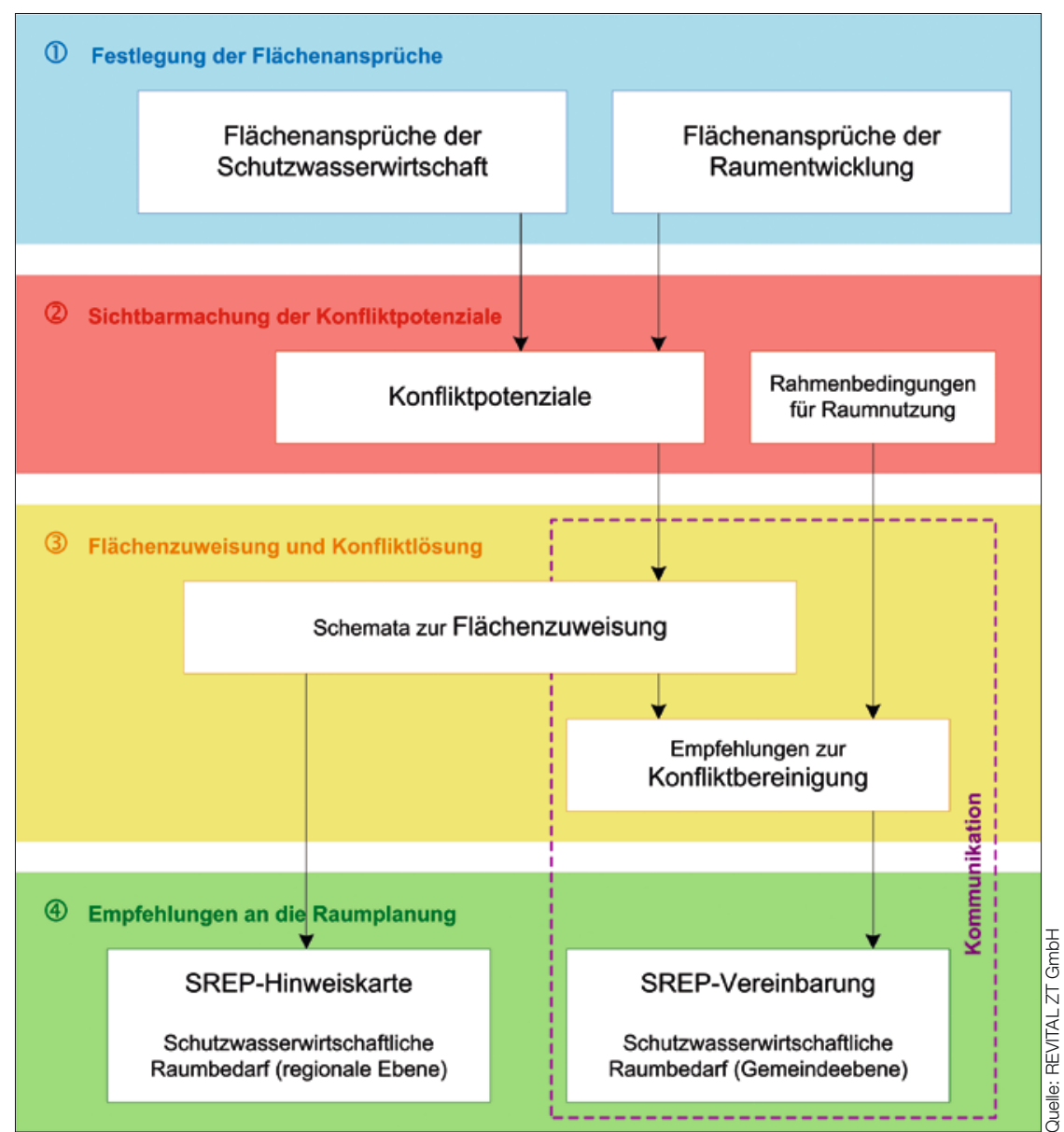

Abb. 1: Übersicht über die Planungsschritte des SREP. 

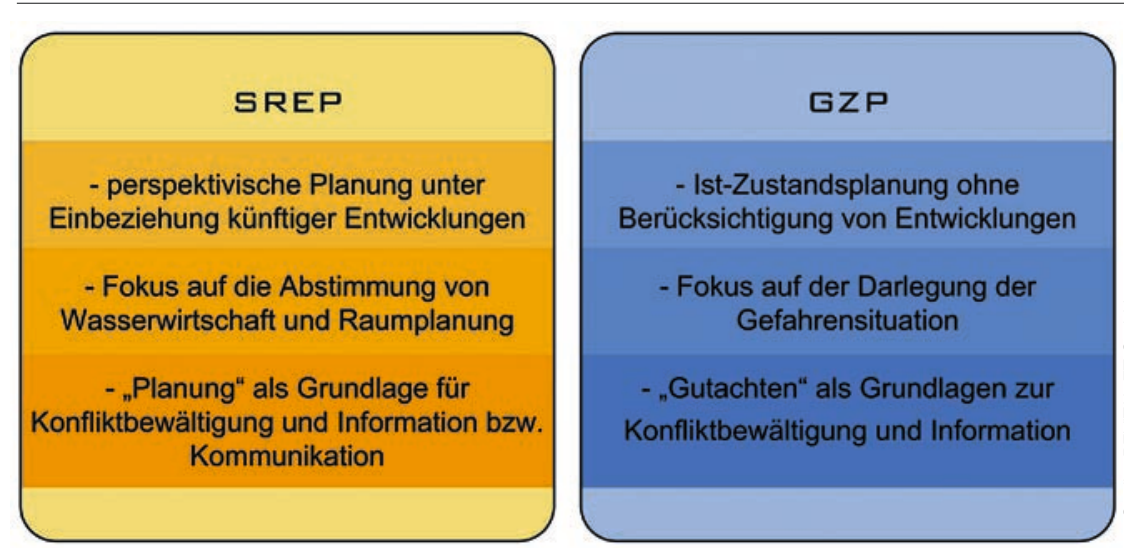

Abb. 2: Gegenüberstellung Gefahrenzonenplan und SREP.

ökologische und erholungsfunktionelle Vorhaben als „Bedarfsflächen“ ein. Die Zonenausweisung des SREP ist somit auf künftige Entwicklung ausgerichtet. Zwar bietet auch die „Blaue Zone“ des Gefahrenzonenplans (=wasserwirtschaftliche Bedarfszone) die Möglichkeit, Flächen für wasserwirtschaftliche Maßnahmen auszuweisen, sie werden jedoch derzeit in Kärnten nicht in Anspruch genommen, da die Bundeswasserbauverwaltung Kärnten Ist-Zustand und Planung strikt auseinanderhält. (Abb. 2)

\subsection{Implementierung des SREP in bestehende Systeme}

Die Ergebnisse des SREP können theoretisch in alle bestehenden raumplanerischen Instrumente (sektorale Programme, überörtliche Raumplanungen, Flächenwidmungspläne, Bebauungspläne) implementiert werden und damit Vorgaben für künftige Planungen bilden. Darüber hinaus, so hat sich gezeigt, bildet der SREP auch eine geeignete Basis für Katastropheneinsatzpläne (Alarmpläne) und Kosten-Nutzenanalysen von Hochwasserschutzmaßnahmen. Entscheidend ist, dass die Öffentlichkeit bei der Erstellung des SREP intensiv eingebunden wird (z. B. in Form von Flussplattformen), um die Akzeptanz bei den BürgerInnen in den betroffenen Gemeinden zu erlangen. Österreichweit gesehen sind Impulse seitens der Raumplanung wie beispielsweise das in Tirol vorgesehene Raumordnungsprogramm für Hochwasserabfluss- und Hochwasserrückhalteräume - zu begrüßen. Entscheidend dabei ist - wie auch beim SREP - die Zusammenarbeit zwischen Raumplanung und Wasserwirtschaft sowie eine einheitliche Vorgangsweise im Planungsablauf. Der in Kärnten entwickelte SREP-
Leitfaden ${ }^{3}$ kann dabei als Orientierung dienen (vgl. Amt der Kärntner Landesregierung, 2009).

\subsection{Einleitung und Vorgehensweise}

Das Projekt „Landwirtschaft und Hochwasser" der Bundesanstalt für Agrarwirtschaft war Teil des Arbeitspakets Raumplanung im Rahmen von FloodRisk II (vgl. Wagner et al., 2008). Die Bundesanstalt für Agrarwirtschaft hat in diesem Projekt onsbewertung landwirtschaftlicher Flächen, das im Interreg III B Projekt ILUP (Wagner, 2007) entwickelt wurde - den Beitrag landwirtschaftlicher Flächen zum Hochwasserschutz sowie deren Hochwasserempfindlichkeit im regionalen Maßstab klassifiziert. Damit können zielgerichtet mögliche Maßnahmen zur Verbesserung der Situation vorgeschlagen und deren ökonomische Bedeutung abgeschätzt werden (Wagner et al., 2008). Als Grundlagen dienen neben der digitalen Bodenkarte, der digitalen Hochwasserrisikozonenausweisung und den INVEKOSFlächennutzungsdaten auch Arbeiten des Institutes für Kulturtechnik und Bodenwasserhaushalt. Richtwerte und Deckungsbeiträge der Bundesanstalt für Agrarwirtschaft fließen in die ökonomische Abschätzung der Maßnahmenkombinationen ein. Die Flächenbewertung wurde

\footnotetext{
Bezugsadresse: Amt der Kärntner Landesregierung, Abteilung 18 - Wasserwirtschaft, Flatschacher Straße 70, A-9020 Klagenfurt, Tel.: +43 50536-31802 oder +43 50536-31828, E-Mail: post.abt18@ktn.gv.at; Internet: http://wasser.ktn.gv.at
}

\section{Die Wechselwirkungen zwischen landwirtschaftlicher Nutzung und dem Hochwassergeschehen} aufbauend auf einem System der Funkti- modellhaft im Gemeindegebiet Seitenstetten in Niederösterreich durchgeführt.

\subsection{Hochwasserschutzfunktionen landwirtschaftlicher Flächen}

Ein natürlicher Beitrag zur Hochwasserprävention landwirtschaftlicher Flächen ergibt sich aus den naturräumlichen $\mathrm{Ge}$ gebenheiten. Die Bodeneigenschaften, Klimabedingungen und Geländeeigenschaften bestimmen den Oberflächenabfluss aus der Fläche, der zusätzlich von der jeweiligen Nutzung der Fläche (Kulturart, Fruchtart, Bearbeitungsverfahren) beeinflusst wird. Als Indikatoren der natürlichen Hochwasserprävention werden die Erosionsgefahr (Strauss, 2007) und die nutzbare Feldkapazität (Murer et al., 2004) herangezogen und mit der landwirtschaftlichen Nutzung in GIS Bearbeitungen überlagert.

Der Einfluss landwirtschaftlicher Nutzung wurde überwiegend aus Bearbeitungen von Klaghofer (2003) Illgen (2000) und Krimly und Dabbert (2007) abgeleitet. Sehr deutlich kommt in den Untersuchungen eine Abstufung der landwirtschaftlichen Nutzung in drei Kategorien heraus: die günstigsten Werte für den Wasserrückhalt weist Grünlandnutzung auf, innerhalb der Ackernutzung ist zwischen Früchten mit durchschnittlichem Oberflächenabfluss bzw. Wasserretentionsvermögen und damit auch Erosionsrisiko und solchen mit erhöhtem Risiko zu unterscheiden. Mittels einer dreistufigen Portfolio-Punktebewertung werden alle landwirtschaftlichen Flächen einzeln klassifiziert. Ein hoher Beitrag zur Hochwasserprävention liegt vor, wenn z.B. ebene Flächen mit speicherfähigen Böden und günstigen Niederschlagsverhältnissen als Grünland genutzt werden. Hingegen ist bei einer Ackernutzung im Allgemeinen mit größeren Oberflächenabflusswerten zu rechnen. Speziell bei bestimmten Ackerreihenfrüchten erhöht sich das Risiko eines starken Oberflächenabflusses, z. B. bei Früchten mit einem späten Bestandesschluss und damit langer Zeit an offener Bodenfläche sowie später Ernte, die eine Zwischenbegrünung erschwert, wie z.B. Mais, Sonnenblumen, Zuckerrüben. Zusätzlich können stärkere Hangneigungen, ungünstige Bodeneigenschaften und zeitlich ungünstige Wetterereignisse zu hohen Wasserabflussmengen führen. Im Projekt war gefordert, Aussagen auf Ebene der Gewässereinzugsgebiete zu erarbeiten, die Prioritäten für die Regionalplanung aufzeigen. 


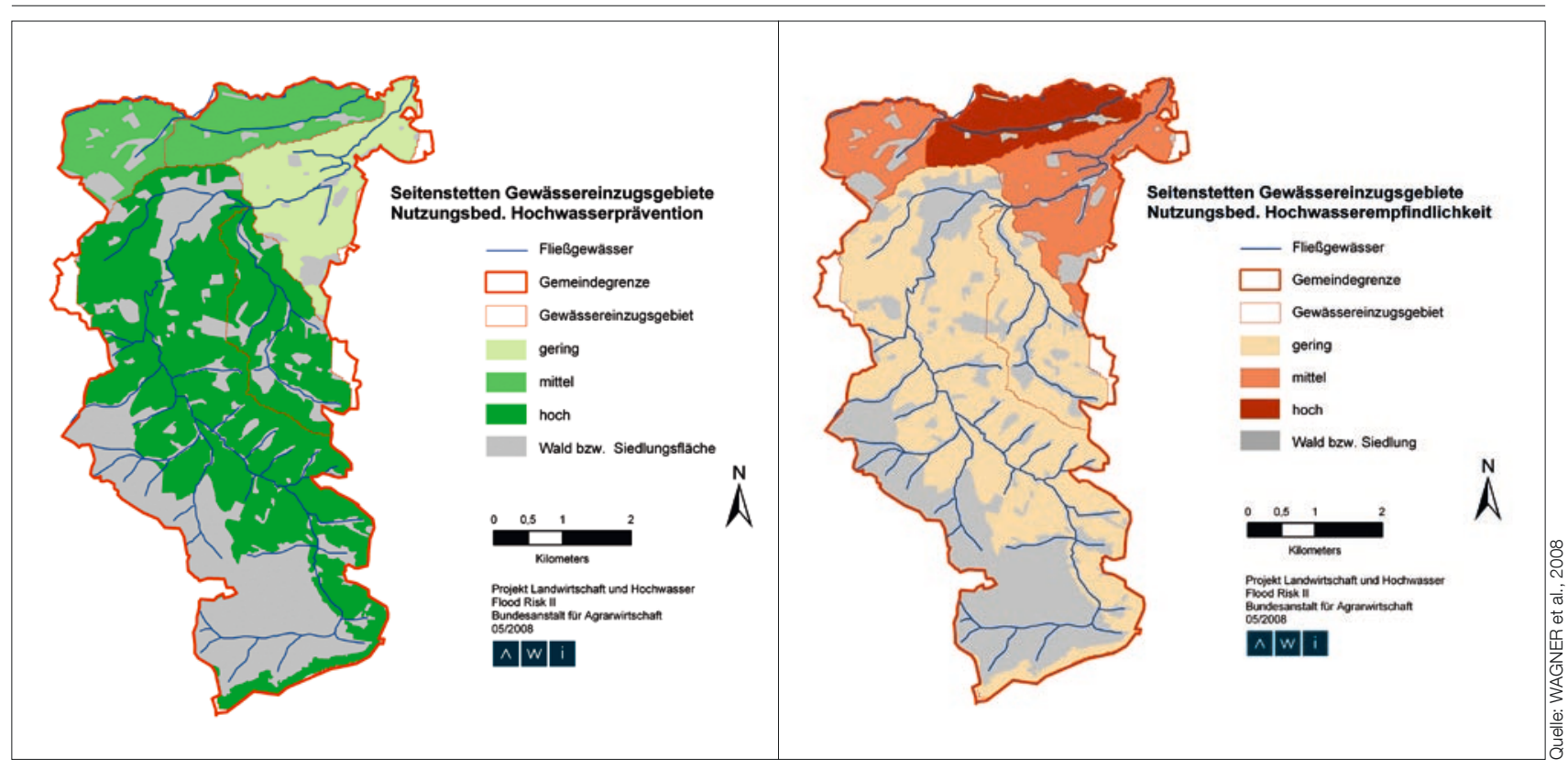

Abb. 3: Hochwasserprävention (linke Karte) und Hochwasserrisiko (rechte Karte) auf landwirtschaftlichen Flächen in Seitenstetten.

Bei der Bewertung der Hochwasserempfindlichkeit wird zusätzlich die Überflutungshäufigkeit der landwirtschaftlichen Flächen berücksichtigt, um risikobehaftete Flächen zu identifizieren. In der vorliegenden Beispielsgemeinde Seitenstetten wurde modellhaft die in eHORA zur Verfügung stehende Hochwasserrisikozonierung verwendet (http://gis.lebensministerium. at/eHORA). Bei einer konkreten Anwendung des Bewertungsmodells muss mit Experten abgeklärt werden, welche Überflutungshäufigkeiten für diesen Zweck den lokalen Geschehnissen am besten gerecht werden. Aufgrund der meist günstigen sonstigen Naturraumeigenschaften in den Überflutungszonen sind es oft genau diese Flächen, wo höhere Anteile an Ackerrisikofrüchten (bezüglich des Oberflächenab- flusses) angebaut werden. Zudem sind dies aber gleichzeitig auch jene Ackerfrüchte, die einen hohen Deckungsbeitrag abwerfen. Mit einer geänderten Fruchtfolge kann man daher den Oberflächenabfluss positiv beeinflussen und gleichzeitig die ökonomischen Schäden im Hochwasserfall verringern, der Landwirt hat aber im Nichtüberflutungsfall ökonomische Nachteile hinzunehmen. (Abb. 3)

\section{3. Ökonomische Bewertung von Nutzungsänderungen}

Maßnahmen, die den Boden- und Wasserrückhalt auf landwirtschaftlichen Flächen verbessern (vgl. dazu Wagner et al., 2008), reichen von unterschiedlichen Bearbeitungstechniken wie Mulch- oder Di-

\section{TABELLE 1}

ÖPUL Maßnahmen mit Wirkung auf den Boden- und Wasserrückhalt

\begin{tabular}{llll} 
Maßnahme & Bodenrückhalt & Wasserrückhalt & Kosten, $\mathbf{\epsilon} / \mathbf{h a}$ \\
\hline $\begin{array}{l}\text { Umweltgerechte } \\
\text { Bewirtschaftung }\end{array}$ & gering & gering & $85-110$ \\
\hline $\begin{array}{l}\text { Erosionsschutz } \\
\text { Dauerkulturen }\end{array}$ & hoch & hoch & $125-300$ \\
\hline $\begin{array}{l}\text { Ackerflächen- } \\
\text { Zwischenfruchtbau }\end{array}$ & hoch & gering & $130-190$ \\
\hline Ackerrandstreifen & hoch & gering & $16-120$ \\
\hline Mulch- und Direktsaat & hoch & mittel & 40 \\
\hline Untersaat bei Mais & hoch & mittel & 50 \\
\hline $\begin{array}{l}\text { Stilllegung in } \\
\text { Projektgebieten }\end{array}$ & hoch & hoch & $300-1.000$ \\
\hline Quelle: eigene Berechnungen & & & \\
\hline
\end{tabular}

rektsaat über Zwischenfruchtanbau oder Umstieg auf weniger risikoreiche Fruchtarten bis zur Umwandlung von Acker in Grünland bzw. zur gänzlichen Aufgabe der Landwirtschaft (vgl. Tab. 1).

Je nach Ertragssituation, ErzeugerInnenpreisen, Betriebssituation und Arbeitsverfahren entstehen durch hochwasserpräventive Nutzungsänderungen unterschiedliche Nachteile für den Landwirt. Diese sind für jedes Projekt zu ermitteln, wobei auch auf die betrieblichen Möglichkeiten der einzelnen LandwirtInnen eingegangen werden muss.

\subsection{Maßnahmen für einen verbesserten Hochwasserschutz und deren Kosten}

Für die Gemeinde Seitenstetten wurden beispielhaft jene Gewässereinzugsgebiete als Maßnahmengebiete herangezogen, die insgesamt nur einen geringen Beitrag zur Hochwasserprävention liefern und die eine hohe Hochwasserempfindlichkeit aufweisen. Aus den GIS-Flächenbilanzen ist abzuleiten, auf wie vielen und auch auf welchen Flächen Nutzungsänderungen empfehlenswert sind. In Summe würde dies für Seitenstetten bedeuten, auf 110 ha von Silomais auf Grünland, auf 22 ha von Körnermais auf Futtergetreide mit Zwischenfruchtanbau umzusteigen und auf 44 ha Ackerflächen Zwischenfrüchte einzusäen.

Die Kosten für diese Art der Bewirtschaftungsumstellung belaufen sich insgesamt auf rund $€ 44.000$,- jährlich (durch- 
schnittlich €250,-/ha Maßnahmenfläche). Damit würden in dem jetzt mit hoher Hochwasserempfindlichkeit bewerteten Einzugsgebiet die Einstufung auf mittel gesenkt werden. Gleichzeitig würde das Einzugsgebiet mit geringer Hochwasserprävention auf die Einstufung hoch angehoben werden und damit ein besserer Beitrag zum Schutz von Unterliegern (auch von Siedlungsflächen) geleistet werden. Wie hoch dieser Beitrag zum vermehrten Wasserrückhalt konkret ist, müsste im Anwendungsfall berechnet werden, um diesen in das Wassermanagementkonzept für ein Gewässereinzugsgebiet einzubeziehen. Diesen Kosten könnte man Alternativen gegenüberstellen, wie z. B. die Bau- und Erhaltungskosten von Schutzanlagen für Siedlungen oder auch die geschätzten Kosten eines einfachen Schadensausgleiches im Schadensfall, je nach Eintrittswahrscheinlichkeit.

\subsection{Fazit}

Die hier erarbeiteten Grundlagen tragen zum Verständnis der Multifunktionalität landwirtschaftlicher Flächen und zu einer Prioritätensetzung im regionalen Maßstab bei. Eine möglichst objektive Einschätzung der ökonomischen Auswirkungen einer angepassten Flächennutzung auf die Landwirtschaft soll Entscheidungen über Maßnahmenpakete zur Hochwasserprävention und zur Schadensminimierung erleichtern. Es wird deutlich, dass die Landwirtschaft gewisse Beitrage zur Hochwasserprävention liefern kann und diese auch ökonomisch durchaus realisierbar erscheinen.

Die Umsetzung der vorgeschlagenen Maßnahmen kann mit verschiedenen Instrumenten und auf unterschiedlichen Ebenen erfolgen, die politisch zu bestimmen wären. $\mathrm{Zu}$ den hoheitlichen, vom Staat geregelten Instrumenten zählen Gesetzgebung, Steuern, Abgaben und Subventionen. $\mathrm{Zu}$ den privaten Instrumenten zählen vertragliche Vereinbarungen (z.B. Nutzungsverträge, Flächenkauf und -pacht), Fonds und Stiftungen sowie handelbare Rechte. Die öffentliche Hand kann im Rahmen der Privatwirtschaftsverwaltung beispielsweise Nutzungsverträge mit GrundeigentümerInnen abschließen (z. B. Vertragshochwasserschutz), Überschwemmungsgebiete aufkaufen oder spezielle Entschädigungsfonds für Hochwasserereignisse einrichten.

Im Rahmen der Hoheitsverwaltung könnte beispielsweise eine „hochwasserverträgliche Landbewirtschaftung “ als zu-

sätzliche Cross Compliance Bestimmung aufgenommen werden. Dazu wären jedoch vorab österreichweite Analysen über die aktuelle Landbewirtschaftung in Einzugs- und Überschwemmungsgebieten und die Ableitung von allgemein anerkannten Kriterien zur hochwasserverträglichen Landbewirtschaftung nötig. Zu überdenken wäre auch eine Einführung neuer bzw. die Erweiterung bestehender Förderprogramme in der ländlichen Entwicklung. Je nach Dringlichkeit der Maßnahmen ist aber zu bedenken, dass die Beteiligung an solchen Programmen auf freiwilliger Basis erfolgt und daher die tatsächliche Wirkung auch von nicht steuerbaren Gegebenheiten, wie z. B. der nationalen und internationalen Preisentwicklung bei landwirtschaftlichen Erzeugnissen oder den Energiepreisen abhängig ist.

\section{Schlussfolgerungen}

Der Artikel stellt aus unterschiedlicher Perspektive die Anforderungen an neue Instrumente des Hochwasserrisikomanagements sowie innovative Ansätze zur Umsetzung einer hochwasserangepassten Raumnutzung dar. Eine vollständige Freihaltung aller potentiellen Gefährdungsbereiche von baulichen Intensivnutzungen wird auch künftig, vor allem in Bereichen mit knappem Dauersiedlungsraum bei gleichzeitig großer Siedlungsdynamik, nicht möglich sein. Die Rolle der Raumplanung wird weiterhin

\section{LITERATUR}

Amt der Kärntner Landesregierung, Abteilung 18 - Wasserwirtschaft (2009) Der Schutzwasserwirtschaftliche Raumentwicklungsplan SREP. Leitfaden, Klagenfurt. Eberstaller J, Berger H, Eberstaller-Fleischanderl D, Gabriel H, Haidvogl G, Kirnbauer R, Seebacher F, Seher W, Stelzhammer, M (2009) Integrative Gewässerentwicklung und interkommunaler Lastenausgleich, Flächenbedarf und -ausgleich für Hochwasserschutz, Siedlungsentwicklung und Ökologie. Studie im Auftrag des Amtes der Niederösterreichischen Landesregierung, Gruppe Wasser, Abteilung WA 3 im Rahmen von FloodRisk II, Wien.

Wien. $P$, Simon A (2003) Sichern und Wiederherstellen von Hochwasserrückhalteflächen. Forschungsbericht im Auftrag des Umweltbundesamtes Deutschland, UBA Texte 34-03, Berlin. Heiland P (2002) Vorsorgender Hochwasserschutz durch Raumordnung, interregionale Kooperation und ökonomischer Lastenausgleich. Verein zur Förderung des Institutes WAR (Hrsg.), Darmstadt.

Illgen M (2000) Überprüfung von Standard-Abflussbeiwerten durch Niederschlag-Abfluss-Simulation, Diplom Arbeit am Fachgebiet Siedlungswasserwirtschaft der Universität Kaiserslautern.

Kanonier A (2005) Naturgefahren im österreichischen Raumordnungsrecht. In: Österreichische Raumordnungskonferenz (ÖROK) (Hrsg.): darin bestehen, jene verbleibenden zusammenhängenden Bereiche zu sichern, die als Hochwasserabfluss- und -rückhalteräume sowie zur Abdeckung des flussmorphologischen Raumbedarfs unbedingt erforderlich sind. Dabei wird der Schwerpunkt auf perspektivische Planungsansätze, Koordination und Kooperation der verschiedenen PlanungsträgerInnen, die Anwendung finanzieller Ausgleichsmaßnahmen sowie die Einbindung der betroffenen LandnutzerInnen zu legen sein. Die breite Ausrichtung der Beiträge zum Arbeitspaket Raumplanung macht deutlich, dass Hochwasserrisikomanagement nicht durch einzelne Disziplinen und Gebietskörperschaften gewährleistet werden kann, sondern als interdisziplinär wahrzunehmende, gesamtgesellschaftliche Aufgabe betrachtet werden muss.

\section{Korrespondenz}

Ass.Prof. DI Dr. Walter Seher

Institut für Raumplanung und Ländliche Neuordnung

Universität für Bodenkultur Wien

Peter Jordan Straße 82, A-1190 Wien

walter.seher@boku.ac.at

DI Dr. Jürgen Eberstaller

ezb - TB Eberstaller GmbH

Schopenhauerstraße 82/12, A-1180 Wien

eberstaller@ezb-fluss.at

DI Klaus Michor

REVITAL Ziviltechniker GmbH

Nußdorf 71, A-9990 Nußdorf-Debant

office@revital-zt.com

DI Klaus Wagner

Bundesanstalt für Agrarwirtschaft

Marxergasse 2, A-1030 Wien

klaus.wagner@awi.bmlfuw.gv.at
Präventiver Umgang mit Naturgefahren in der Raumordnung, 81-114, ÖROK Schriftenreihe Nr. 168, Wien.

Klaghofer E (2003) Hochwasser und Landnutzung, in: Schriftenreihe des Bundesamtes für Wasserwirtschaft 19, 60-69, Wien.

Krimly T, Dabbert St (2007) Landwirtschaftlicher Hochwasserschutz, Institut für Landwirtschaftliche Betriebslehre, Universität Hohenheim, Stuttgart.

Murer E et al. (2004) Die nutzbare Feldkapazität der mineralischen Böden der landwirtschaftlichen Nutzfläche Österreichs. Schriftenreihe des Bundesamtes für Wasserwirtschaft, Band 20, Wien, S. 72-78.

Seher W, Berger H (2009) Praktische Umsetzung künftiger Strategien risikoarmer Raumnutzung, Projektteil Interkommunale Kooperation. Studie im Auftrag des Bundesministeriums für Verkehr, Innovation und Technologie (BMVIT) im Rahmen von FloodRisk II, Wien.

Strauss P (2007) Flächenhafter Bodenabtrag durch Wasser. In: Hydrologischer Atlas Österreichs, 3. Lieferung, Kartentafel 8.4, Lebensministerium Wien.

Wagner K (2007) Landwirtschaftliche Grundlagen für eine Integrierte Regionalplanung.

Schriftenreihe der Bundesanstalt für Agrarwirtschaft Nr. 48, Wien.

Schaft Nr. 48, Wien.

(2008) Projektforschungsbericht Landwirtschaft und Hochwasser. Bundesanstalt für Agrarwirtschaft, Wien. 